\title{
Synthesis and Antimicrobial Activities of 5-Arylidene-thiazolidine-2,4-dione Derivatives
}

\author{
Ivanildo Mangueira da Silva, ${ }^{1}$ João da Silva Filho, ${ }^{2}$ \\ Priscila Brandão Gomes da Silva Santiago, ${ }^{1}$ Micalyne Soares do Egito, ${ }^{1}$ \\ Carlos André de Souza, ${ }^{2}$ Frederico Leite Gouveia, ${ }^{1}$ Rafael Matos Ximenes, ${ }^{2}$ \\ Kêsia Xisto da Fonseca Ribeiro de Sena, ${ }^{2}$ Antonio Rodolfo de Faria, ${ }^{1}$ \\ Dalci José Brondani, ${ }^{1}$ and Julianna Ferreira Cavalcanti de Albuquerque ${ }^{1,2}$ \\ ${ }^{1}$ Department of Pharmacy, Federal University of Pernambuco, Avenida Professor Moraes Rego 1235, Cidade Universitária, \\ 50670-901 Recife, PE, Brazil \\ ${ }^{2}$ Department of Antibiotics, Federal University of Pernambuco, Avenida Professor Moraes Rego 1235, Cidade Universitária, \\ 50670-901 Recife, PE, Brazil
}

Correspondence should be addressed to Julianna Ferreira Cavalcanti de Albuquerque; julianna@ufpe.br

Received 27 February 2014; Revised 14 April 2014; Accepted 15 April 2014; Published 7 May 2014

Academic Editor: Terry K. Smith

Copyright (C) 2014 Ivanildo Mangueira da Silva et al. This is an open access article distributed under the Creative Commons Attribution License, which permits unrestricted use, distribution, and reproduction in any medium, provided the original work is properly cited.

\begin{abstract}
Antibiotic resistance is considered one of the world's major public health concerns. The main cause of bacterial resistance is the improper and repeated use of antibiotics. To alleviate this problem, new chemical substances against microorganisms are being synthesized and tested. Thiazolidines are compounds having many pharmacological activities including antimicrobial activities. For this purpose some thiazolidine derivatives substituted at position 5 in the thiazolidine nucleus were synthesized and tested against several microorganisms. Using a disc diffusion method, antimicrobial activity was verified against Gram-positive, Gramnegative, and alcohol acid resistant bacteria and yeast. The minimum inhibition concentrations (MIC) and minimum bactericidal concentrations $(\mathrm{MBC})$ were determined. All derivatives showed antimicrobial activity mainly against Gram-positive bacteria, with MIC values ranging from 2 to $16 \mu \mathrm{g} / \mathrm{mL}$.
\end{abstract}

\section{Introduction}

Thiazolidine is a class of compounds which merit special attention because it belongs to a group of substances with activity in medicinal chemistry. This nucleus is associated with antibacterial, antifungal, antiviral, antituberculosis, anticancer, and antiparasite biological activities [1-4]. The use of new synthetic methods and structure-activity relationship studies has made possible a broad study of new drugs with different actions. The computational search for possible mechanisms of 4-thiazolidinones anticancer activity has been studied together with the medical chemistry which permits the optimization of existing drugs [5].
Currently, very important groups of heterocyclic compounds such as thiazolidine-2,4-dione, 2-imino-4-thiazolidinone, 4-thioxo-thiazolidine-2,4-dione, and 2-thioxo-1,3-thiazolidine-4-one (rhodanine) have attracted the attention of researchers in their search for new agents with specific pharmacological properties [6-14].

Multiple antibiotic resistant bacteria represent a challenge in the treatment of infections. It is imperative, therefore, that new substances with antimicrobial properties be found to fight these microorganisms [15]. To be considered a bacterium resistant to a certain antibiotic, the microorganism should be able to grow in vitro when subjected to an inhibitory concentration equal to that obtained in the blood. 
However, the concentration of several antibiotics in the bloodstream can be much lower than that achieved by the same antibiotic in other body tissues or fluids. Thus, a bacterium could be "resistant" to a certain antibiotic when it is present in the bloodstream but "sensitive" when it is in the urinary tract or vice versa [16].

Bacteria become resistant to chemotherapeutic agents by three main mechanisms: destruction or inactivation of the drug, prevention of the penetration of the target site within the microbe, and alteration of drug target sites. There may be variations in these mechanisms [17]. With increasing bacterial resistance to antibiotics, attention has become focused on the development of new derivatives to be used as antimicrobial therapy in infection control [18]. Antibiotics are substances produced synthetically by bacteria and fungi with the function of suppressing the growth of microorganisms [19]. Currently, new antibiotics are needed for the treatment of multidrug resistant bacteria. The clinical use of new drugs has decreased since the 1980s, due to a reduction in the discovery of new, more efficient, and less toxic drugs by pharmaceutical companies around the world. Other research groups are worried about the rise in recurrence of many infectious diseases and the lack of new drugs and development of new antimicrobial products in the face of increasing resistance to existing agents [20].

The literature reports on the results of a number of biological activities when the substituents and their positions on the thiazolidine ring are changed [21]. In this case, medicinal chemistry is an important aid in the discovery of new active molecules using small heterocyclic rings to increase the biological activity of certain nuclei [22]. Due to the importance of the core of the thiazolidine ring, eleven compounds have been synthesized by our research group, some products already known and others unknown by introducing arylidene groups at the position 5 of the thiazolidine ring in order to test the antimicrobial activity of each compound against nine different microorganisms.

\section{Material and Methods}

2.1. Chemistry. The chemical reagents were supplied by Sigma-Aldrich (USA) and were used without further purification. Purity of the compounds was checked using thin layer chromatography (TLC) plates (silica gel G) in the appropriated system for each compound. The spots were located with short $(254 \mathrm{~nm}) /$ long $(365 \mathrm{~nm})$ UV wavelength.

All melting points were measured in a capillary tube on a Quimis apparatus. Infrared spectra of $1 \% \mathrm{KBr}$ pellets were recorded using a Bruker IFS66 spectrometer. ${ }^{1} \mathrm{H}$ NMR and ${ }^{13} \mathrm{C}$ NMR spectra were measured on a VARIAN VNMRS 400$\mathrm{MR}$, using $400 \mathrm{MHz}$ for ${ }^{1} \mathrm{H}$ and $75.4 \mathrm{MHz}$ for ${ }^{13} \mathrm{C}$ in $\mathrm{CDCl}_{3}$ and acetone- $\mathrm{d}_{6}$ maintained at $25^{\circ} \mathrm{C}$ using $\mathrm{Me}_{4} \mathrm{Si}$ (TMS) as an internal standard. The chemical shifts were reported in $\delta$ units and the coupling constants $(J)$ were reported in hertz. The following abbreviations were used to indicate the peak multiplicity: $\mathrm{s}$ (singlet), $\mathrm{d}$ (doublet), dd (double doublet), $\mathrm{t}$ (triplet), and $\mathrm{m}$ (multiplet). $\mathrm{C}, \mathrm{H}, \mathrm{N}$, and $\mathrm{S}$ analyses were performed with a Carlo Erba elemental analyzer, model

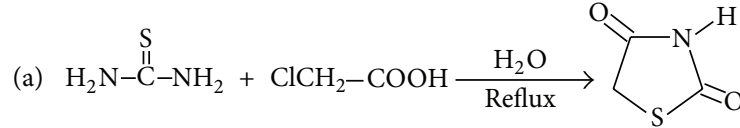

(1)

(b)<smiles>[R]c1cccc(/C=C2\SC(=O)NNC2=O)c1</smiles>

(1)
$\left.\mathrm{R}=\mathbf{2 a}) 3-\mathrm{OCH}_{3}, 4-\mathrm{OH} ; \mathbf{2 b}\right) 2,4-\mathrm{Cl}_{2}$; 2c) $3,4-\mathrm{Cl}$; 2d) $4-\mathrm{OH}$;

2e) $3-\mathrm{OCH}_{3}$; 2f) $3-\mathrm{CH}_{3}$; 2g) $3-\mathrm{NO}_{2}$; 2h) $4-\mathrm{NO}_{2}$; 2i) $3-\mathrm{Cl}$

Scheme 1: Substituent in the synthesis of 5-arylidene-thiazolidine2,4-dione (2a-i).

EA1108. Mass spectra were recorded on a Varian MAT 711 spectrometer at an electron impact of $70 \mathrm{eV}$. The synthesized compounds are shown in Scheme 1, showing the respective substituent.

2.2. Procedure for Preparation of the Synthesis of Thiazolidine2,4-dione (1). Compound (1) (thiazolidine-2,4-dione) was synthesized by refluxing monochloroacetic acid and thiourea in water. This compound was synthesized according to a published procedure [16]. Molecular formula $\mathrm{C}_{3} \mathrm{H}_{3} \mathrm{O}_{2} \mathrm{NS}$; yield 78\%; mp $118-120^{\circ} \mathrm{C}$; Rf 0.48 (0.9: $\left.0.1 \mathrm{CHCl}_{3} / \mathrm{MeOH}\right)$. Recrystallization: water.

2.3. General Method for the Synthesis of 5-Arylidene-thiazolidine-2,4-dione $(\mathbf{2} \mathbf{a}-\mathbf{i})$. The compound $(\mathbf{2} \mathbf{a}-\mathbf{i})$ was synthesized from a mixture of thiazolidine-2,4-dione (1) $(2.5 \mathrm{~g}$, $21.36 \mathrm{mmol})$, aldehyde (21.36 mmol), piperidine $(14.11 \mathrm{mmol})$, and ethanol $(150 \mathrm{~mL})$. The reaction mixture was heated under reflux and continuously stirred for a period of 8-9 h. The course of the reaction was monitored by TLC. The reaction mixture was poured into water and acidified with acetic acid. The resulting precipitate was filtered off and recrystallized from acetic acid to give (2a-i).

2.4. Microbiological Activity. Gram-positive, Gram-negative, and alcohol acid resistant bacteria and yeast were selected for the examination of antimicrobial activity in vitro. For the evaluation of the antimicrobial activity, first a disc diffusion test was used to screen the antimicrobial activity of all compounds. The compounds that had inhibition zones greater than $10 \mathrm{~mm}$ of diameter were submitted to a second test for the determination of minimum inhibitory concentration (MIC) and minimum bactericidal concentration (MBC). The tests were performed as follows.

2.4.1. Disk Diffusion Method. Antimicrobial activity was evaluated by disc diffusion method according to Bauer et al. [23] 
against Gram-positive bacteria (Staphylococcus aureusDAUFPE 01, Micrococcus luteus-DAUFPE 06, Bacillus subtilis-DAUFPE 16, and Enterococcus faecalis-DAUFPE 138), Gram-negative bacteria (Pseudomonas aeruginosaDAUFPE 39, Escherichia coli-DAUFPE 224, and Serratia marcescens-DAUFPE 398), acid alcohol resistant bacteria (Mycobacterium smegmatis-DAUFPE 71), and yeast (Candida albicans-DAUFPE 1007) obtained from the culture collection of the Department of Antibiotics at the Federal University of Pernambuco (UFPE), Brazil.

Paper discs (Whatman number 2) with $6 \mathrm{~mm}$ diameters were impregnated with $20 \mu \mathrm{L}$ of a $15,000 \mu \mathrm{g} / \mathrm{mL}$ solution of the synthesized compounds and dissolved in DMSO. The discs were then placed in medium sown with one of the microorganisms. The following standard drugs were used as controls: ketoconazole (Neoquímica, Brazil), for yeast and cefalexin (Eurofarma, Brazil) and kanamycin (Centro de Controle e Produtos para Diagnósticos-CECON, Brazil) for bacteria. The tests were carried out 3 times, with the results expressed (in millimeters) as mean \pm SEM of the diameters of the inhibition zones formed around the discs. The negative control test was carried out with DMSO soaked discs.

\subsubsection{Minimum Inhibitory Concentration (MIC) and Mini-} mum Bactericidal Concentration (MBC). A multiwell plate (96 wells) was used to determine the minimum inhibitory concentration (MIC) following the criteria adopted by the Clinical and Laboratory Standards Institute (CLSI) [24]. Tests were carried out in Müeller-Hinton broth and Sabouraud dextrose broth (Difco, USA) at pH 7.4 and the twofold serial dilution technique was applied. A 1,280 $\mu \mathrm{g} / \mathrm{mL}$ stock solution was prepared from the product. A standardized suspension of microorganisms was prepared for use with a 0.5 tube on the McFarland scale. The plate was incubated for 18 hours and thereafter an indicator dye resazurin (Sigma-Aldrich, USA) was applied to show if there was microbial growth in the well. MIC was determined as the concentration of the last well where there was no microbial growth. From this experiment, the content of the wells was sown on plates with MüellerHinton agar medium to establish the minimum bactericidal concentration (MBC), which is the concentration where there is no colony growth. All analyses were performed in triplicate.

\section{Result and Discussion}

\subsection{Synthesis}

5-(3-Methoxy-4-hydroxy-arylidene)-thiazolidine-2,4-dione (2a). Yield 70\%; mp $260^{\circ} \mathrm{C}$; Rf $0.50\left(\mathrm{CHCl}_{3} / \mathrm{MeOH} 9: 1\right)$. Recrystallization: ethanol; IR ( $\left.\mathrm{KBr} 1 \%, v_{\max } \mathrm{cm}^{-1}\right) 1566$ $(\mathrm{C}=\mathrm{C}) ; 1730-1670(\mathrm{C}=\mathrm{O}) .{ }^{1} \mathrm{H}$ NMR $\left(\mathrm{CDCl}_{3}, 300 \mathrm{MHz}, \delta\right.$ ppm): 8.48 (s $1 \mathrm{H}, \mathrm{NH}$ ); 7.71 (s $1 \mathrm{H}, \mathrm{CH}=) ; 3.77$ (s $3 \mathrm{H}, \mathrm{OCH}_{3}$ ); $6.02(\mathrm{~s} 1 \mathrm{H}, \mathrm{OH}) .6 .92\left(\mathrm{~s} \mathrm{1H}_{(2)}\right) ; 6.86\left(\mathrm{~d} \mathrm{1H}_{(5)} J=8.33\right) ; 6.91(\mathrm{~d}$ $\left.1 \mathrm{H}_{(6)} J=8.34\right) ;{ }^{13} \mathrm{C}$ NMR (Acetone- $\left.\mathrm{d}_{6}, 75.4 \mathrm{MHz}, \delta \mathrm{ppm}\right)$ : $169.81\left(\mathrm{C}=\mathrm{O}_{2}\right) ; 166.89\left(\mathrm{C}=\mathrm{O}_{4}\right) ; 119.55\left(\mathrm{C}_{5}\right.$, heterocycle $)$; $143.28(\mathrm{CH}=) ; 56.50\left(\mathrm{OCH}_{3}\right) ; 129.20\left(\mathrm{C}_{1}\right) ; 112.58\left(\mathrm{C}_{2}\right), 148.06$ $\left(\mathrm{C}_{3}\right) ; 144.76\left(\mathrm{C}_{4}\right) ; 115.56\left(\mathrm{C}_{5}\right) ; 122.30\left(\mathrm{C}_{6}\right)$. Anal. Calcd. for
$\mathrm{C}_{11} \mathrm{H}_{9} \mathrm{NO}_{4} \mathrm{~S}: \mathrm{C},(52.58 \%) ; \mathrm{H},(3.61 \%) ; \mathrm{N},(5.57 \%)$. Found: C, $53.01 \%$; H, 3.83\%; N, 5.25\%. HRMS ${ }^{+}$: calcd 251.0252; found 251.0253 .

5-(2,4-Dichloro-arylidene)-thiazolidine-2,4-dione (2b). Yield 65\%; mp 203 ${ }^{\circ} \mathrm{C}$; Rf $0.51\left(\mathrm{CHCl}_{3} / \mathrm{MeOH} 9.6: 0.4\right)$. Recrystallization: ethanol; IR (KBr 1\%, $\left.v_{\max } \mathrm{cm}^{-1}\right) 1570(\mathrm{C}=\mathrm{C})$; 1737$1675(\mathrm{C}=\mathrm{O}) .{ }^{1} \mathrm{H}$ NMR $\left(\mathrm{CDCl}_{3}, 300 \mathrm{MHz}, \delta \mathrm{ppm}\right): 8.50$ (s $1 \mathrm{H}$, $\mathrm{NH}$ ); 7.35 (s $1 \mathrm{H}, \mathrm{CH}=) ; 7.55$ (s $1 \mathrm{H}, \mathrm{H}_{(3)} 7.44$ (d 1H, $\mathrm{H}_{(5)} J=$ 7.90); $7.39\left(\mathrm{~d} 1 \mathrm{H}\left(\mathrm{H}_{(6)} \mathrm{J}=7.90\right) .{ }^{13} \mathrm{C}\right.$ NMR (Acetone- $\mathrm{d}_{6}$, $75.4 \mathrm{MHz}, \delta \mathrm{ppm}): 169.85\left(\mathrm{C}=\mathrm{O}_{2}\right) ; 166.86\left(\mathrm{C}=\mathrm{O}_{4}\right) ; 120.20\left(\mathrm{C}_{5}\right.$ heterocycle); $140.23(\mathrm{CH}=) ; 133.61\left(\mathrm{C}_{1}\right) ; 134.84\left(\mathrm{C}_{2}\right) ; 129.52$ $\left(\mathrm{C}_{3}\right) ; 134.93\left(\mathrm{C}_{4}\right) ; 127.52\left(\mathrm{C}_{5}\right) ; 135.80\left(\mathrm{C}_{6}\right)$. Anal. Calcd. for $\mathrm{C}_{10} \mathrm{H}_{5} \mathrm{Cl}_{2} \mathrm{NO}_{2} \mathrm{~S}$ : C, 43.81\%; H, 1.84\%; N, 5.11\%. Found: C, $44.01 \%$; H, 1.96\%; N, 5.05\%. HRMS ${ }^{+}$: calcd 272.9418; found 272.9419 .

5-(3,4-Dichloro-arylidene)-thiazolidine-2,4-dione (2c). Yield $59 \%$; mp $174^{\circ} \mathrm{C}$; Rf $0.50\left(\mathrm{CHCl}_{3} / \mathrm{MeOH} 9.5: 0.5\right)$. Recrystallization: ethanol; IR (KBr 1\%, $\left.v_{\max } \mathrm{cm}^{-1}\right) 1571(\mathrm{C}=\mathrm{C})$; $1735-$ $1676(\mathrm{C}=\mathrm{O}) .{ }^{1} \mathrm{H}$ NMR $\left(\mathrm{CDCl}_{3}, 300 \mathrm{MHz}, \delta \mathrm{ppm}\right): 8.50(\mathrm{~s} 1 \mathrm{H}$, $\mathrm{NH}) ; 7.77$ (s $1 \mathrm{H}, \mathrm{CH}=) ; 7.52$ (s $1 \mathrm{H}, \mathrm{H}_{(2)} ; 7.38\left(\mathrm{~d} 1 \mathrm{H}, \mathrm{H}_{(5)}\right.$ $J=8.91) ; 7.52\left(\mathrm{~d} 1 \mathrm{H}\left(\mathrm{H}_{(6)} J=8.92\right) .{ }^{13} \mathrm{C}\right.$ NMR (Acetone$\left.\mathrm{d}_{6}, 75.4 \mathrm{MHz}, \delta \mathrm{ppm}\right): 169.86\left(\mathrm{C}=\mathrm{O}_{2}\right) ; 166.89\left(\mathrm{C}=\mathrm{O}_{4}\right) ; 119.17$ $\left(\mathrm{C}_{5}\right.$ heterocycle $) ; 143.13(\mathrm{CH}=) ; 134.42\left(\mathrm{C}_{1}\right) ; 129.49\left(\mathrm{C}_{2}\right) ; 133.15$ $\left(\mathrm{C}_{3}\right) ; 131.79\left(\mathrm{C}_{4}\right) ; 130.71\left(\mathrm{C}_{5}\right) ; 127.26\left(\mathrm{C}_{6}\right)$. Anal. Calcd. for $\mathrm{C}_{10} \mathrm{H}_{5} \mathrm{Cl}_{2} \mathrm{NO}_{2} \mathrm{~S}$ : C, 43.81\%; H, $1.84 \%$; N, 5.11\%. Found: C, 43.91\%; H, 1.97\%; N, 5.07\%. HRMS ${ }^{+}$: calcd 272.9418; found 272.9419 .

5-(4-Hidroxy-arylidene)-thiazolidine-2,4-dione (2d). Yield $45 \%$; mp 149- $150^{\circ} \mathrm{C}$; Rf $0.49\left(\mathrm{CH}_{2} \mathrm{Cl}_{2} / \mathrm{Hex} .5\right.$ : 5). Recrystallization: methanol; IR ( $\left.\mathrm{KBr} 1 \%, \nu_{\max } \mathrm{cm}^{-1}\right) 1570(\mathrm{C}=\mathrm{C})$; 1734-1677 (C=O). ${ }^{1} \mathrm{H}$ NMR $\left(\mathrm{CDCl}_{3}, 300 \mathrm{MHz}, \delta \mathrm{ppm}\right): 8.49$ (s $1 \mathrm{H}, \mathrm{NH}) ; 7.54$ (s $1 \mathrm{H}, \mathrm{CH}=) ; 6.40$ (s $1 \mathrm{H}, \mathrm{OH}) ; 7.31$ (d $1 \mathrm{H}$, $\left.\mathrm{H}_{(2)}-\mathrm{H}_{(6)} \mathrm{J}=8.65\right) ; 7.21\left(\mathrm{~d} 1 \mathrm{H}, \mathrm{H}_{(3)}-\mathrm{H}_{(5)} \mathrm{J}=8.65\right) .{ }^{13} \mathrm{C}$ NMR (Acetone- $\left.\mathrm{d}_{6}, 75.4 \mathrm{MHz}, \delta \mathrm{ppm}\right): 169.86\left(\mathrm{C}=\mathrm{O}_{2}\right) ; 166.89$ $\left(\mathrm{C}=\mathrm{O}_{4}\right) ; 119.55\left(\mathrm{C}_{5}\right.$ heterocycle $) ; 140.25(\mathrm{CH}=) ; 129.15\left(\mathrm{C}_{1}\right)$; $129.15\left(\mathrm{C}_{2}\right)-\left(\mathrm{C}_{6}\right) ; 115.96\left(\mathrm{C}_{3}\right)-\left(\mathrm{C}_{5}\right) ; 157.71\left(\mathrm{C}_{4}\right)$. Anal. Calcd. for $\mathrm{C}_{10} \mathrm{H}_{7} \mathrm{NO}_{3} \mathrm{~S}$ : C, 54.29\%; H, 3.19\%; N, 6.33\%. Found: C, $54.93 \%$; H, 3.23\%; N, 6.11\%. HRMS ${ }^{+}$: calcd 221.0147; found 272.0148

5-(4-Methoxy-arylidene)-thiazolidine-2,4-dione (2e). Yield 40\%; mp 210-212 ${ }^{\circ} \mathrm{C}$; Rf $0.48\left(\mathrm{CHCl}_{3} / \mathrm{MeOH}\right.$ 9.6:0.4). Recrystallization: $\mathrm{CHCl}_{3}$; IR ( $\left.\mathrm{KBr} 1 \%, \nu_{\max } \mathrm{cm}^{-1}\right) 1575$ $(\mathrm{C}=\mathrm{C}) ; 1734-1676 \quad(\mathrm{C}=\mathrm{O}) .{ }^{1} \mathrm{H}$ NMR $\left(\mathrm{CDCl}_{3}, 300 \mathrm{MHz}\right.$, $\delta \mathrm{ppm}): 8.49$ (s $1 \mathrm{H}, \mathrm{NH}) ; 7.74(\mathrm{~s} 1 \mathrm{H}, \mathrm{CH}=) ; 3.65$ (s $3 \mathrm{H}$, $\left.\mathrm{OCH}_{3}\right) ; 7.35\left(\mathrm{~d} \mathrm{1H}, \mathrm{H}_{(2)}-\mathrm{H}_{(6)} J=8.65\right) ; 6.75\left(\mathrm{~d} \mathrm{1H}\left(\mathrm{H}_{(3)}{ }^{-}\right.\right.$ $\left.\mathrm{H}_{(5)} J=8.65\right) .{ }^{13} \mathrm{C}$ NMR (Acetone- $\left.\mathrm{d}_{6}, 75.4 \mathrm{MHz}, \delta \mathrm{ppm}\right)$ : $169.86\left(\mathrm{C}=\mathrm{O}_{2}\right) ; 166.89\left(\mathrm{C}=\mathrm{O}_{4}\right) ; 119.50 \quad\left(\mathrm{C}_{5}\right.$ heterocycle $)$; $142.55(\mathrm{CH}=) ; 55.67\left(\mathrm{OCH}_{3}\right) ; 128.42\left(\mathrm{C}_{1}\right) ; 129.37\left(\mathrm{C}_{2}\right)-\left(\mathrm{C}_{6}\right)$; $114.32\left(\mathrm{C}_{3}\right)-\left(\mathrm{C}_{5}\right) ; 160.31\left(\mathrm{C}_{4}\right)$. Anal. Calcd. for $\mathrm{C}_{11} \mathrm{H}_{9} \mathrm{NO}_{3} \mathrm{~S}$ : C, 56.16\%; H, 3.86\%; N; 5.95\%. Found: C, 56.98\%; H, 3.99\%; $\mathrm{N}, 6.45 \% . \mathrm{HRMS}^{+}$: calcd 235.0303; found 235.0303 .

5-(3-Methyl-arylidene)-thiazolidine-2,4-dione (2f). Yield 52\%; mp 195-196 C; Rf 0.50 (Hex./Ethyl acetate 8.5:1.5). 
Recrystallization: acetic acid; IR ( $\left.\mathrm{KBr} 1 \%, \nu_{\max } \mathrm{cm}^{-1}\right) 1573$ $(\mathrm{C}=\mathrm{C}) ; 1730-1739(\mathrm{C}=\mathrm{O}) ;{ }^{1} \mathrm{H}$ NMR $\left(\mathrm{CDCl}_{3}, 300 \mathrm{MHz}, \delta\right.$ ppm): 8.49 (s $1 \mathrm{H}, \mathrm{NH}) ; 7.73$ (s $1 \mathrm{H}, \mathrm{CH}=) ; 2.36$ (s, $3 \mathrm{H} \mathrm{CH}_{3}$ ); 7.25 ( $\left.\mathrm{s} 1 \mathrm{H} \mathrm{H}_{(2)}\right) ; 6.95$ ( $\left.\mathrm{d} 1 \mathrm{H} \mathrm{H}_{(4)} J=7.71\right) ; 7.31(\mathrm{t}, 2 \mathrm{H}$ $\left.\mathrm{H}_{5}, J=7.72\right) ; 7.31\left(\mathrm{~d} 1 \mathrm{H} \mathrm{H}_{6} J=7.52\right) .{ }^{13} \mathrm{C} \mathrm{NMR}$ (Acetone$\left.\mathrm{d}_{6}, 75.4 \mathrm{MHz}, \delta \mathrm{ppm}\right): 169.86\left(\mathrm{C}=\mathrm{O}_{2}\right) ; 167.89\left(\mathrm{C}=\mathrm{O}_{4}\right) ; 119.55$ $\left(\mathrm{C}_{5}\right.$ heterocycle); $143.18(\mathrm{CH}=) ; 21.20 \mathrm{CH}_{3} ; 136.18\left(\mathrm{C}_{1}\right)$; $128.93\left(\mathrm{C}_{2}\right) ; 133.87\left(\mathrm{C}_{3}\right) ; 126.60\left(\mathrm{C}_{4}\right) ; 129.51\left(\mathrm{C}_{5}\right) ; 125.93$ $\left(\mathrm{C}_{6}\right)$. Anal. Calcd. for $\mathrm{C}_{11} \mathrm{H}_{9} \mathrm{NO}_{2} \mathrm{~S}$ : C, 60.26\%; $\mathrm{H}, 4.14 \%$; , 6.39\%. Found: C, 60.66\%; H, 4.45\%; N, 6.11\%; $\mathrm{HRMS}^{+}$: calcd 219.0354; found 272.0353 .

5-(3-Nitro-arylidene)-thiazolidine-2,4-dione (2g). Yield 80\%; mp $250^{\circ} \mathrm{C}$; Rf $0.51\left(\mathrm{CHCl}_{3} / \mathrm{MeOH} 9.6: 0.4\right)$. Recrystallization: acetic acid. IR (KBr 1\%, $\left.v_{\max } \mathrm{cm}^{-1}\right) 1571(\mathrm{C}=\mathrm{C}) ; 1740-1667$ $(\mathrm{C}=\mathrm{O}) ;{ }^{1} \mathrm{H} \mathrm{NMR}\left(\mathrm{CDCl}_{3}, 300 \mathrm{MHz}, \delta \mathrm{ppm}\right): 8.49$ (s $\left.1 \mathrm{H}, \mathrm{NH}\right)$; 7.37 (s $1 \mathrm{H}, \mathrm{CH}=)$; 8.35 ( $\left.\mathrm{s} 1 \mathrm{H} \mathrm{H}_{(2)}\right) 7.92\left(\mathrm{~d} 1 \mathrm{H} \mathrm{H}_{(4)}, J=\right.$

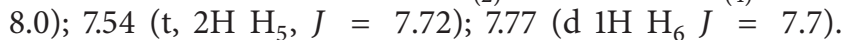
${ }^{13} \mathrm{C}$ NMR (Acetone- $\left.\mathrm{d}_{6}, 75.4 \mathrm{MHz}, \delta \mathrm{ppm}\right): 169.86\left(\mathrm{C}=\mathrm{O}_{2}\right)$; $166.88\left(\mathrm{C}=\mathrm{O}_{4}\right) ; 119.55\left(\mathrm{C}_{5}\right.$ heterocycle $) ; 143.65(\mathrm{CH}=) ; 136.25$ $\left(\mathrm{C}_{1}\right) ; 123.06\left(\mathrm{C}_{2}\right) ; 148.74\left(\mathrm{C}_{3}\right) ; 122.14\left(\mathrm{C}_{4}\right) ; 130.16\left(\mathrm{C}_{5}\right) ; 131.91$ $\left(\mathrm{C}_{6}\right)$. Anal. Calcd. for $\mathrm{C}_{10} \mathrm{H}_{6} \mathrm{~N}_{2} \mathrm{O}_{4} \mathrm{~S}: \mathrm{C}, 48.00 \%$; $\mathrm{H}, 2.42 \%$; , $11.20 \%$. Found: C, 48.35\%; H, 2.1\%; N, 11.01\%; $\mathrm{HRMS}^{+}$: calcd 250.0048; found 250.0051 .

5-(4-Nitro-arylidene)-thiazolidine-2,4-dione (2h). Yield 69\%; mp 220-223 ${ }^{\circ} \mathrm{C}$; Rf $0.52\left(\mathrm{CHCl}_{3} / \mathrm{MeOH} 9.6: 0.4\right)$. Recrystallization: THF; IR (KBr 1\%, $\left.\nu_{\max } \mathrm{cm}^{-1}\right) 1575$ (C=C); 1734-1676 $(\mathrm{C}=\mathrm{O}) .{ }^{1} \mathrm{H} \mathrm{NMR}\left(\mathrm{CDCl}_{3}, 300 \mathrm{MHz}, \delta \mathrm{ppm}\right): 8.49$ (s $\left.1 \mathrm{H}, \mathrm{NH}\right)$; 7.74 (s $1 \mathrm{H}, \mathrm{CH}=) ; 7.63$ (d $\left.1 \mathrm{H}, \mathrm{H}_{(2)}-\mathrm{H}_{(6)} J=8.65\right) ; 8.09$ (d $1 \mathrm{H}\left(\mathrm{H}_{(3)}-\mathrm{H}_{(5)} J=8.65\right) .{ }^{13} \mathrm{C}$ NMR (Acetone- $\mathrm{d}_{6}, 75.4 \mathrm{MHz}, \delta$ ppm): $169.86\left(\mathrm{C}=\mathrm{O}_{2}\right) ; 166.89\left(\mathrm{C}=\mathrm{O}_{4}\right) ; 119.50\left(\mathrm{C}_{5}\right.$ heterocycle $)$; $142.55(\mathrm{CH}=) ; 141.50\left(\mathrm{C}_{1}\right) ; 129.37\left(\mathrm{C}_{2}\right)-\left(\mathrm{C}_{6}\right) ; 124.13\left(\mathrm{C}_{3}\right)-$ $\left(\mathrm{C}_{5}\right)$; $147.21\left(\mathrm{C}_{4}\right)$; Anal. Calcd. for $\mathrm{C}_{10} \mathrm{H}_{6} \mathrm{~N}_{2} \mathrm{O}_{4} \mathrm{~S}$ : C, 48.00\%; H, 2.42\%; N, 11.20\%. Found: C, 48.35\%; H, 2.1\%; N, 11.01\%; $\mathrm{HRMS}^{+}$: calcd 250.0048; found 250.0049.

5-(3-Chloro-arylidene)-thiazolidine-2,4-dione (2i). Yield 54\%; mp 208-209 ${ }^{\circ}$; Rf 0.46 (Hexane/Ethyl acetate $4: 1$ ). Recrystallization: ethyl acetate; IR ( $\left.\mathrm{KBr} 1 \%, v_{\max } \mathrm{cm}^{-1}\right) 1582(\mathrm{C}=\mathrm{C})$; 1729-1672 (C=O). ${ }^{1} \mathrm{H}$ NMR $\left(\mathrm{CDCl}_{3}, 300 \mathrm{MHz}, \delta \mathrm{ppm}\right): 8.48$ (s $1 \mathrm{H}, \mathrm{NH}) ; 7.77$ (s $1 \mathrm{H}, \mathrm{C}=\mathrm{H}) ; 7.34\left(\mathrm{~s} 1 \mathrm{H}_{(2)}\right) ; 7.18\left({\mathrm{~d} 1 \mathrm{H}_{(4)}}\right.$ $(J=8.07)$; 7.36 (t $2 \mathrm{H}_{(5)}(J=8.07) ; 7.36\left({\mathrm{~d} 1 \mathrm{H}_{(6)}} J=2.27\right)$; ${ }^{13} \mathrm{C}$ NMR (Acetone- $\left.\mathrm{d}_{6}, 75.4 \mathrm{MHz}, \delta \mathrm{ppm}\right): 169.86\left(\mathrm{C}=\mathrm{O}_{2}\right)$; $166.46\left(\mathrm{C}=\mathrm{O}_{4}\right) ; 119.55\left(\mathrm{C}_{5}\right.$ heterocycle $) ; 143.09(\mathrm{CH}=) ; 135.81$ $\left(\mathrm{C}_{1}\right) ; 127.72\left(\mathrm{C}_{2}\right) ; 133.85\left(\mathrm{C}_{3}\right) ; 128.62\left(\mathrm{C}_{4}\right) ; 130.25\left(\mathrm{C}_{5}\right) ; 125.78$ $\left(\mathrm{C}_{6}\right)$. Anal. Calcd. for $\mathrm{C}_{10} \mathrm{H}_{6} \mathrm{ClNO}_{2} \mathrm{~S}$ : C, $50.11 \%$; $\mathrm{H}, 2.52 \% ; \mathrm{N}$, $5.84 \%$. Found: C, 50.55\%; H, 2.67\%; N, 5.57\%. HRMS ${ }^{+}$: calcd 238.9808; found 238.9810 .

The compounds $(\mathbf{2} \mathbf{a}-\mathbf{i})$ were synthesized by Knoevenagel condensation with nine aromatic aldehydes yielding 5-arylidene-thiazolidine-2,4-dione derivatives. The infrared spectrum of these compoundsshowed a strong absorption band of the functional group ranging between 1566 at $1775 \mathrm{~cm}^{-1}$ corresponding to $\mathrm{C}=\mathrm{C}$ and $1775-1676 \mathrm{~cm}^{-1}$ concerning the carbonyl in positions 2 and 4 of the thiazolidine-2,4-dione ring [25]. The molecular structures of the compounds were established by ${ }^{1} \mathrm{H}$ NMR and ${ }^{13} \mathrm{C}$ NMR spectra which exhibited the presence of the signals corresponding to hydrogen and carbons in the molecule. The compound (2a) presented a singlet at 3.77 ppm of 3 hydrogen referent to $\mathrm{OCH}_{3}$ and a singlet at 6.02 concerning the absorption of the $\mathrm{OH}$ group. This substituent also presented absorption at $56.50 \mathrm{ppm}$ of the $\mathrm{OCH}_{3}$ in ${ }^{13} \mathrm{C}$ NMR. The group $\mathrm{OCH}_{3}$ in (2d) presented signal in 3.77 ppm to hydrogen and 56.50 to absorption in ${ }^{13} \mathrm{C}$ NMR. The absorption of hydrogen as a singlet at $3.65 \mathrm{ppm}$ of the $\mathrm{OCH}_{3}$ group at position 4 of arylidene and 55.67 to ${ }^{13} \mathrm{C}$ NMR was sufficient to characterize the compound (2e). The compound (2f) presented absorption as a singlet at $2.36 \mathrm{ppm}$ to hydrogen in methyl group at position 3 and $20.15 \mathrm{ppm}$ to ${ }^{13} \mathrm{C}$ NMR in the molecule. The ${ }^{1} \mathrm{H}$ NMR spectra of compounds showed only one kind of proton absorption for $\mathrm{CH}=$ in the range 7.717.78. According to the literature, this proton absorption is the compound represented by the configuration $Z$, as illustrated by the absorption of the $\mathrm{CH}=$ group. Isomer $(Z)$ appears to be more thermodynamically stable than isomers $E$ [26].

The ${ }^{1} \mathrm{H}$ NMR spectrum of compounds in $\mathrm{CDCl}_{3}-\mathrm{d} 6$ revealed the presence of methyne moiety and the position of the hydrogen of the aromatic group. In addition, the structures were supported by their mass spectrum which revealed a molecular ion peak. The elemental analysis was also important to supplement the characterization of the chemical structure.

3.2. Antimicrobial Activity. The results of the disc diffusion test are shown in Table 1. Most of the synthesized compounds were active against Gram-positive bacteria. MIC and MBC values are shown in Table 2.

Compound (1) thiazolidine-2,4-dione showed no antimicrobial activity against any of the tested microorganisms [12]. When it reacted with aromatic benzaldehydes forming derivatives substituted at position 5, however, all derivatives showed antimicrobial activity. This affirmation is similar to what is in the literature, showing that substitution at position 5 with arylidene groups has an important effect on enhancing the antimicrobial properties [14].

This shows the importance of the dual $\mathrm{CH}=$ bond at position 5, thus introducing a pharmacophore group in the molecule. This group generally influences the specific structural changes in biological effects, stereochemistry, size, and shape of the carbon chain as well as the nature of the other substituent. The compound (2a) with substituents 3$\mathrm{OCH}_{3}$ and 4-OH of the arylidene ring was the most active of all, showing better inhibition for almost all microorganisms tested. This compound inhibited all Gram-positive bacteria tested and, additionally, was the only one that inhibited the growth of Gram-negative bacteria, alcohol acid resistant bacteria, and also the yeast, as shown in Table 1. Generally, 4-thiazolidinediones derivatives have demonstrated antimicrobial activity against Gram-positive and Gram-negative bacteria [14].

Compounds (2b) and (2c), containing the substituents 2,4- $\mathrm{Cl}_{2}$ and 3,4- $\mathrm{Cl}_{2}$, respectively, inhibited all Gram-positive bacteria tested and also the alcohol acid resistant bacteria. 


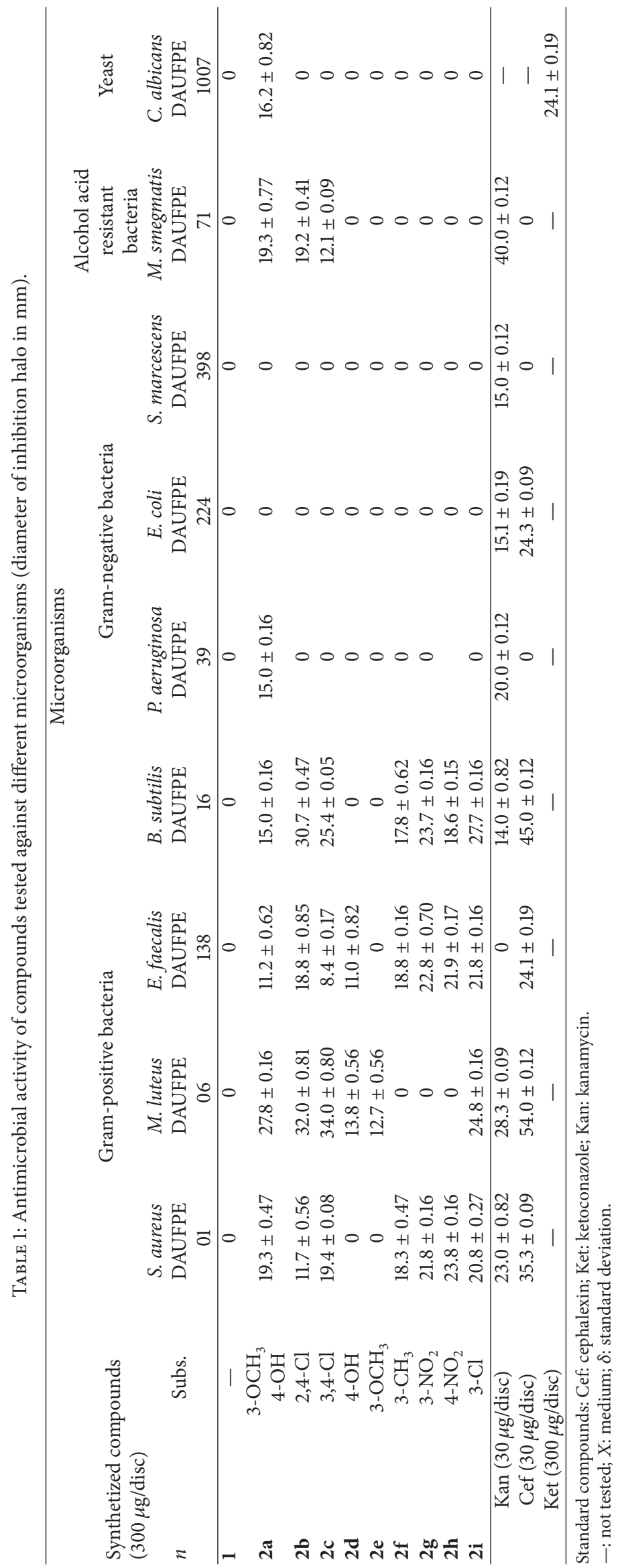




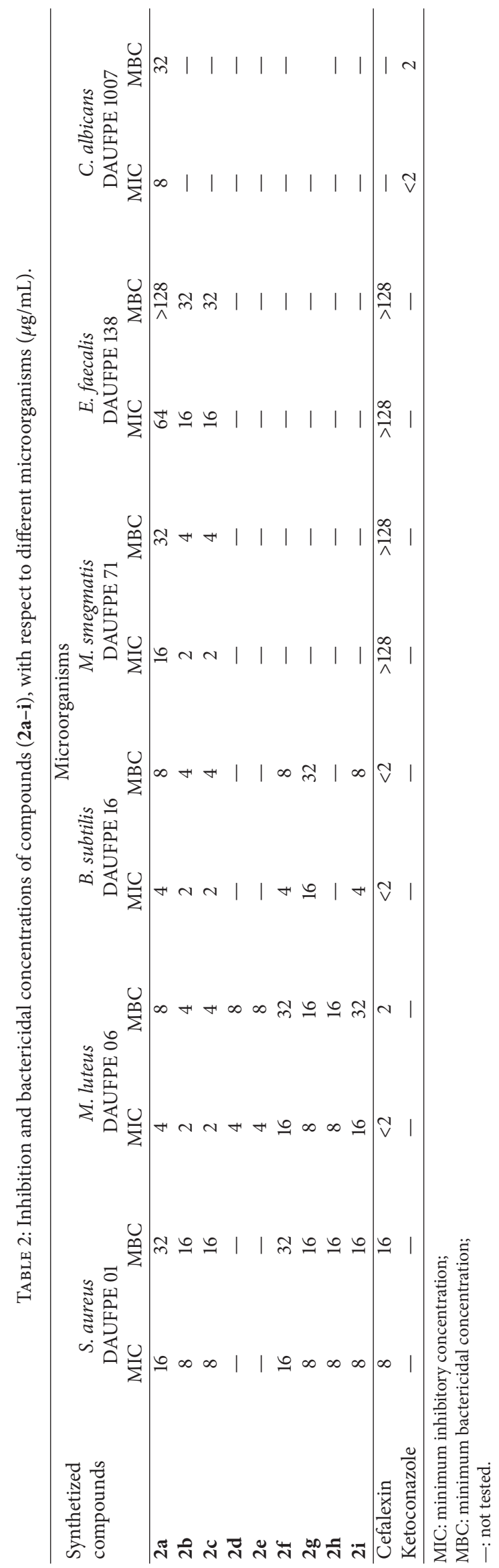


However, compound (2c) showed halos slightly smaller than (2b).

The compound (2d) with 4-OH group was active only for two Gram-positive bacteria, M. Luteus and E. faecalis, while (2e), substituted with $3-\mathrm{OCH}_{3}$, was only active against M. luteus. This fact indicates that the groups $\mathrm{OH}$ and $\mathrm{OCH}_{3}$ separately do not contribute to a significant antimicrobial activity. This fact is reinforced by the activity of compound (2a), which had both groups $\left(3-\mathrm{OCH}_{3}\right.$ and $\left.4-\mathrm{OH}\right)$, against all tested microorganisms, including the Gram-negative bacterium $P$. aeruginosa. The compounds $(\mathbf{2} \mathbf{f}, \mathbf{2 g}$, and $\mathbf{2 h}$ ) were active only for Gram-positive bacteria, except E. faecalis, with halos between $17.8 \pm 0.62$ and $23.8 \pm 0.16 \mathrm{~mm}$. The compound (2i) also inhibited the growth of all Gram-positive bacteria.

In general, all compounds tested were active against Gram-positive bacteria presenting values of 16 and $32 \mu \mathrm{g} / \mathrm{mL}$ for S. aureus and 4 and $8 \mu \mathrm{g} / \mathrm{mL}$ for M. luteus and B. subtilis, for MIC and MBC, respectively. MIC and MBC values for $M$. smegmatis were 16 and $32 \mu \mathrm{g} / \mathrm{mL}$, smaller than the standard drugs. The compounds $(\mathbf{2 b}, \mathbf{2 c}, \mathbf{2 g}, \mathbf{2 h}$, and $\mathbf{2 i})$ showed MIC and MBC values for $S$. aureus near those obtained for the standard drug, cefalexin. The compounds (2b) and (2c) showed MIC and MBC for M. Luteus and B. subtilis also near the standard drug. The results for $M$. smegmatis 16 and $32 \mu \mathrm{g} / \mathrm{mL}$ were lower than the standard Kanamycin $(128 \mu \mathrm{g} / \mathrm{mL})$ showing significant activity in inhibiting these microorganisms. Recent studies with 5-hydroxy-arylidene derivatives showed MIC values higher than $128 \mu \mathrm{g} / \mathrm{mL}$ for two Gram-negative bacteria, E. coli and P. aeruginosa, and $128 \mu \mathrm{g} / \mathrm{mL}$ for E. faecalis and S. aureus [7].

These results showed that these substituents separately did not have significant antimicrobial activity. The groups chlorine, nitro, and methyl at position 3 on the thiazolidine ring seem to favor this activity for other positions [7]. This affirmation reveals that electrons donating or withdrawing groups in molecules increase the lipophilicity of the compounds, which alters the permeability across the bacterial cell membrane [27]. These molecules showed the greatest inhibition among the tested compounds. The compounds $(\mathbf{2 b}, \mathbf{2 c}$, and $\mathbf{2 i})$ containing chlorine atoms in the molecule were active for all Gram-positive bacteria, indicating the power of chlorine in antimicrobial activity.

This demonstrates that the substitution of arylidene at position 5 on the thiazolidine ring enhances the antimicrobial activity of the pharmacophore group in these compounds. The condensation of the compounds 5-arylidenethiazolidine-2,4-dione was crucial to generate considerable activity in the synthesized derivatives [8]. The results of this study demonstrate significant antimicrobial activity for compounds 5-arylidene-thiazolidine-2,4-dione substituted $(2 \mathbf{a}-\mathbf{i})$, with values similar to the drugs tested cephalexin $>128$, confirming the antimicrobial activity of thiazolidine compounds as described in the literature [1, 27]. These compounds probably act on the synthesis of peptidoglycan from the cell wall, which explains the higher antimicrobial effects on Gram-positive bacteria instead of Gram-negative ones $[2,28]$.

\section{Conclusion}

In order to find an antimicrobial agent, nine compounds were synthesized and tested against Gram-positive, Gramnegative, and alcohol acid resistant bacteria and yeast. All compounds were active against all Gram-positive bacteria. One of the compounds was the most active, inhibiting Grampositive, Gram-negative, and alcohol acid resistant bacteria and also the yeast. The other compounds had varied activity between classes of Gram-positive and acid alcohol resistant bacteria. Compounds containing chlorine in the molecule showed the best antibacterial activity, thus demonstrating the power of this atom on the bacteria. The compounds with substituents nitro and methyl also showed significant activity. All the compounds presented the $Z$ configuration. The chemical structures of the compounds were determined by physical methods IR, ${ }^{1} \mathrm{HNMR},{ }^{13} \mathrm{CNMR}$ and mass spectrometry.

\section{Conflict of Interests}

The authors declare that there is no conflict of interests regarding the publication of this paper.

\section{Acknowledgments}

The authors would like to thank the CNPq for financial support and the Fundamental Chemistry Department of Federal University of Pernambuco for the use of the Analytical Center. The English version of this work has been revised by Sidney Pratt, Canadian, B.A., M.A.T. (The Johns Hopkins University), RSAdip (TEFL).

\section{References}

[1] A. Verma and S. K. Saraf, "4-Thiazolidinone-a biologically active scaffold," European Journal of Medicinal Chemistry, vol. 43, no. 5, pp. 897-905, 2008.

[2] C. J. Andres, J. J. Bronson, S. V. D’Andrea et al., "4Thiazolidinones: novel inhibitors of the bacterial enzyme MurB," Bioorganic \& Medicinal Chemistry Letters, vol. 10, no. 8, pp. 715-717, 2000.

[3] M. Tunçbilek and N. Altanlar, "Synthesis and antimicrobial evaluation of some 3-(substituted phenacyl)-5-[4'-(4H-4oxo-1-benzopyran-2-yl)-benzylidene]-2,4- thiazolidinediones," Il Farmaco, vol. 54, no. 7, pp. 475-478, 1999.

[4] O. Devinyak, D. Havrylyuk, B. Zimenkovsky, and R. Lesyk, "Computational search for possible mechanisms of 4thiazolidinones anticancer activity: the power of visualization," Molecular Informatics, vol. 33, no. 3, pp. 216-229, 2014.

[5] T. Tomašić and L. P. Mašič, "Rhodanine as a privileged scaffold in drug discovery," Current Medicinal Chemistry, vol. 16, no. 13, pp. 1596-1629, 2009.

[6] A. M. C. Andrade, W. T. Lima, M. P. A. Rocha et al., "Synthesis and structural study of substituted thioxothiazolidinones and thioxoimidazolidinones," Bollettino Chimico Farmaceutico, vol. 141, no. 6, pp. 428-433, 2002.

[7] T. Tomašić, N. Zidar, A. Kovač et al., "5-benzylidenethiazolidin4-ones as multitarget inhibitors of bacterial Mur ligases," ChemMedChem, vol. 5, no. 2, pp. 286-295, 2010. 
[8] M. S. A. El-Gaby, G. A. M. El-Hag Ali, A. A. El-Maghraby, M. T. Abd El-Rahman, and M. H. M. Helal, "Synthesis, characterization and in vitro antimicrobial activity of novel 2-thioxo-4-thiazolidinones and 4,4' -bis(2-thioxo-4-thiazolidinone-3-yl)diphenylsulfones," European Journal of Medicinal Chemistry, vol. 44, no. 10, pp. 4148-4152, 2009.

[9] R. B. Lesyk, B. S. Zimenkovsky, D. V. Kaminskyy et al., "Thiazolidinone motif in anticancer drug discovery. Experience of DH LNMU medicinal chemistry scientific group," Biopolymers and Cell, vol. 27, no. 2, pp. 107-117, 2011.

[10] A. A. R. Silva, A. J. da Silva Góes, W. T. de Lima, and M. B. de Souza Maia, "Antiedematogenic activity of two thiazolidine derivatives: $N$-tryptophyl-5-(3,5-di-tert-butyl-4hydroxybenzylidene) rhodanine (GS26) and N-tryptophyl-5(3,5-di-tert-butyl-4-hydroxybenzylidene)-2,4-thiazolidinedione (GS28)," Chemical and Pharmaceutical Bulletin, vol. 51, no. 12, pp. 1351-1355, 2003.

[11] T. Tomašić and L. P. Masic, "Prospects for developing new antibacterials targeting bacterial type IIA topoisomerases," Current Topics in Medicinal Chemistry, vol. 14, no. 1, pp. 130-151, 2014.

[12] F. L. Gouveia, R. M. B. de Oliveira, T. B. de Oliveira et al., "Synthesis, antimicrobial and cytotoxic activities of some 5arylidene-4-thioxo-thiazolidine-2-ones," European Journal of Medicinal Chemistry, vol. 44, no. 5, pp. 2038-2043, 2009.

[13] R. Ottanà, R. MacCari, M. L. Barreca et al., "5-Arylidene-2imino-4-thiazolidinones: design and synthesis of novel antiinflammatory agents," Bioorganic and Medicinal Chemistry, vol. 13, no. 13, pp. 4243-4252, 2005.

[14] P. Vicini, A. Geronikaki, K. Anastasia, M. Incerti, and F. Zani, "Synthesis and antimicrobial activity of novel 2thiazolylimino-5-arylidene-4-thiazolidinones," Bioorganic and Medicinal Chemistry, vol. 14, no. 11, pp. 3859-3864, 2006.

[15] W. Tavares, Manual de Antibióticos e Quimioterápicos AntiInfecciosos, Atheneu, São Paulo, Brazil, 3th edition, 2002.

[16] K. Babaoglu, M. A. Page, V. C. Jones et al., "Novel inhibitors of an emerging target in Mycobacterium tuberculosis; substituted thiazolidinones as inhibitors of dTDP-rhamnose synthesis," Bioorganic and Medicinal Chemistry Letters, vol. 13, no. 19, pp. 3227-3230, 2003.

[17] G. J. Tortora and B. R. Funke, Microbiologia, Artes Médicas Sul, Porto Alegre, Brazil, 6th edition, 2003.

[18] O. Bozdağ-Dündar, Ö. Özgen, A. Menteşe et al., "Synthesis and antimicrobial activity of some new thiazolyl thiazolidine-2,4dione derivatives," Bioorganic and Medicinal Chemistry, vol. 15, no. 18, pp. 6012-6017, 2007.

[19] T. Gumbo, "General principles of antimicrobial therapy," in Goodman \& Gilman's The Pharmacological Basis of Therapeutics, L. L. Brunton, B. A. Chabner, and B. C. Knollmann, Eds., McGraw-Hill, 12th edition, 2012.

[20] I. Chopra, C. Schofield, M. Everett et al., "Treatment of healthcare-associated infections caused by Gram-negative bacteria: a consensus statement," The Lancet Infectious Diseases, vol. 8, no. 2, pp. 133-139, 2008.

[21] S. Malik, P. K. Upadhyaya, and S. Miglani, “Thiazolidinediones: a plethro of biological load," International Journal of PharmTech Research, vol. 3, no. 1, pp. 62-75, 2011.

[22] S. Allen, B. Newhouse, A. S. Anderson et al., "Discovery and SAR of trisubstituted thiazolidinones as CCR4 antagonists," Bioorganic and Medicinal Chemistry Letters, vol. 14, no. 7, pp. 1619-1624, 2004.
[23] A. W. Bauer, W. M. Kirby, J. C. Sherris, and M. Turck, "Antibiotic susceptibility testing by a standardized single disk method," The American Journal of Clinical Pathology, vol. 45, no. 4, pp. 493496, 1966.

[24] Clinical and Laboratory Standards Institute (CLSI), Performance Standards for Antimicrobial Disk Susceptibility Tests, 23rd informational supplement M100-S23, Clinical and Laboratory Standards Institute (CLSI), Wayne, Pa, USA, 2013.

[25] M. Tunçbilek, O. Bozdag-Dündar, G. Ayhan-Kilcigil et al., "Synthesis and hypoglycemic activity of some substituted flavonyl thiazolidinedione derivatives-fifth communication: Flavonyl benzyl substituted 2,4-thiazolidinediones," Il Farmaco, vol. 58, no. 1, pp. 79-83, 2003.

[26] G. Bruno, L. Costantino, C. Curinga et al., "Synthesis and aldose reductase inhibitory activity of 5-arylidene-2,4-thiazolidinediones," Bioorganic and Medicinal Chemistry, vol. 10, no. 4, pp. 1077-1084, 2002.

[27] A. Kocabalkanli, Ö. Ates, and G. Ötük, "Synthesis of Mannich bases of some 2, 5-disubstituted 4-thiazolidinones and evaluation of their antimicrobial activities," Archiv der Pharmazie, vol. 334, no. 2, pp. 35-39, 2001.

[28] N. Zidar, T. Tomašić, R. Šink et al., "New 5-benzylidenethiazolidin-4-one inhibitors of bacterial MurD ligase: design, synthesis, crystal structures, and biological evaluation," European Journal of Medicinal Chemistry, vol. 46, no. 11, pp. 5512-5523, 2011. 

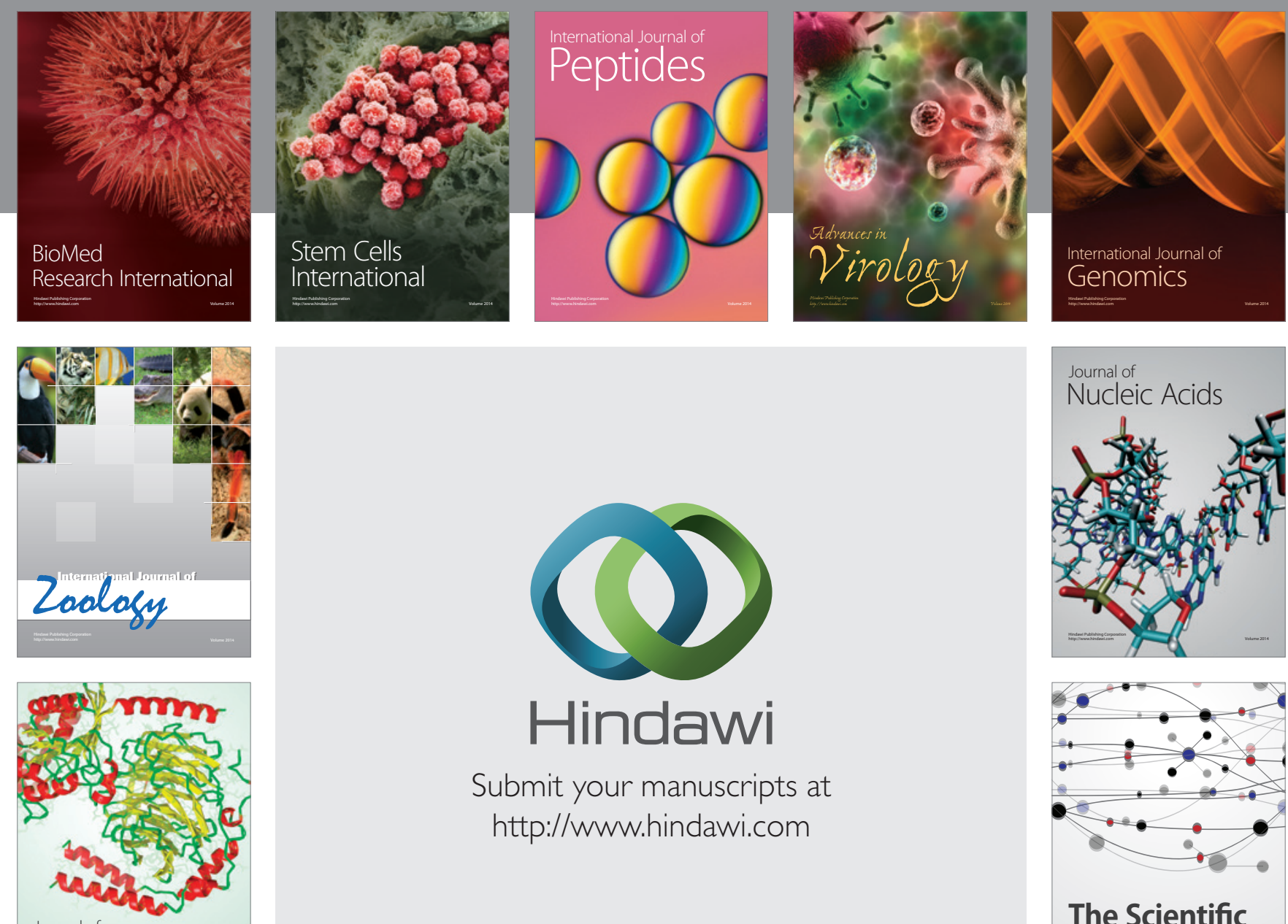

Submit your manuscripts at

http://www.hindawi.com

Journal of
Signal Transduction
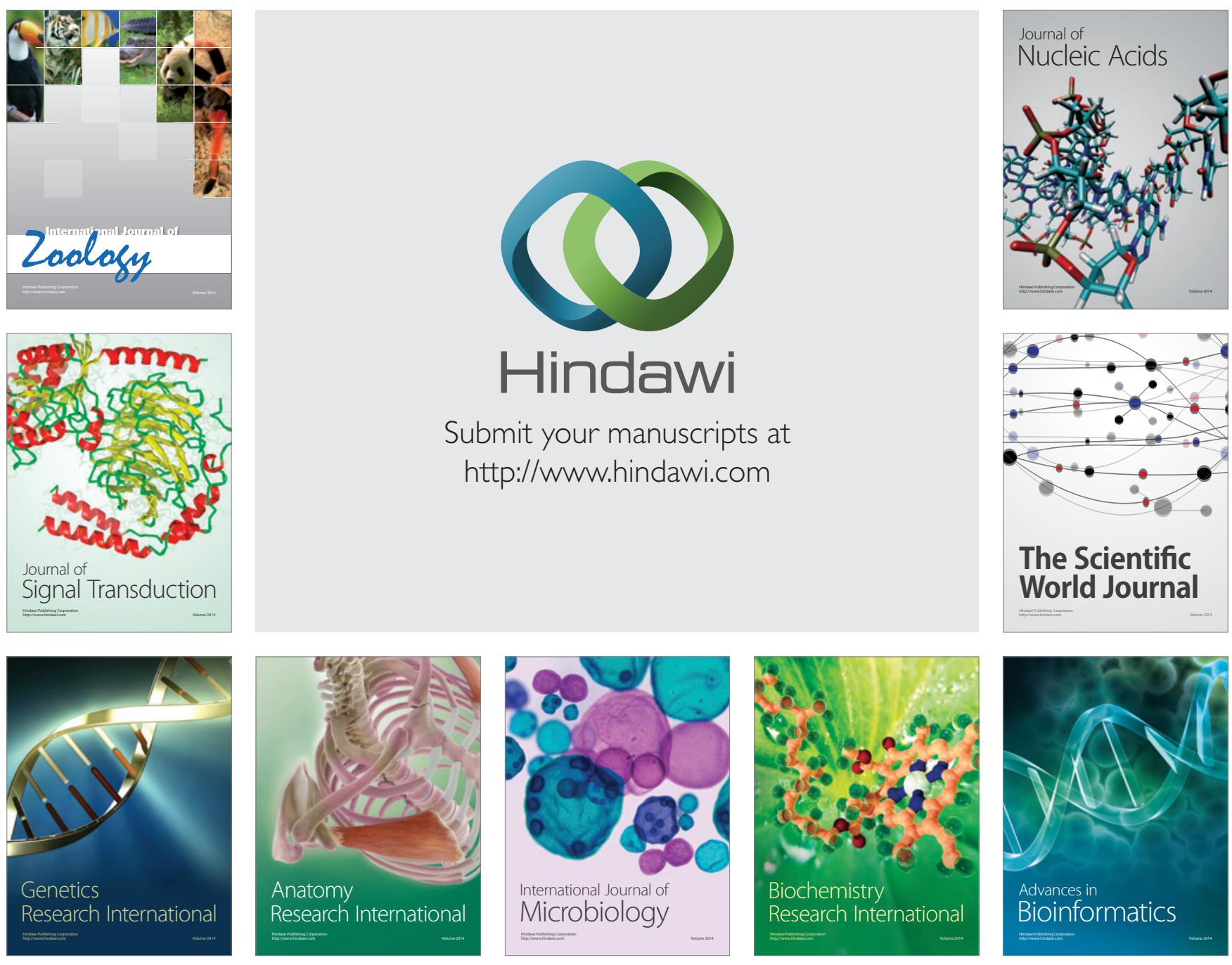

The Scientific World Journal
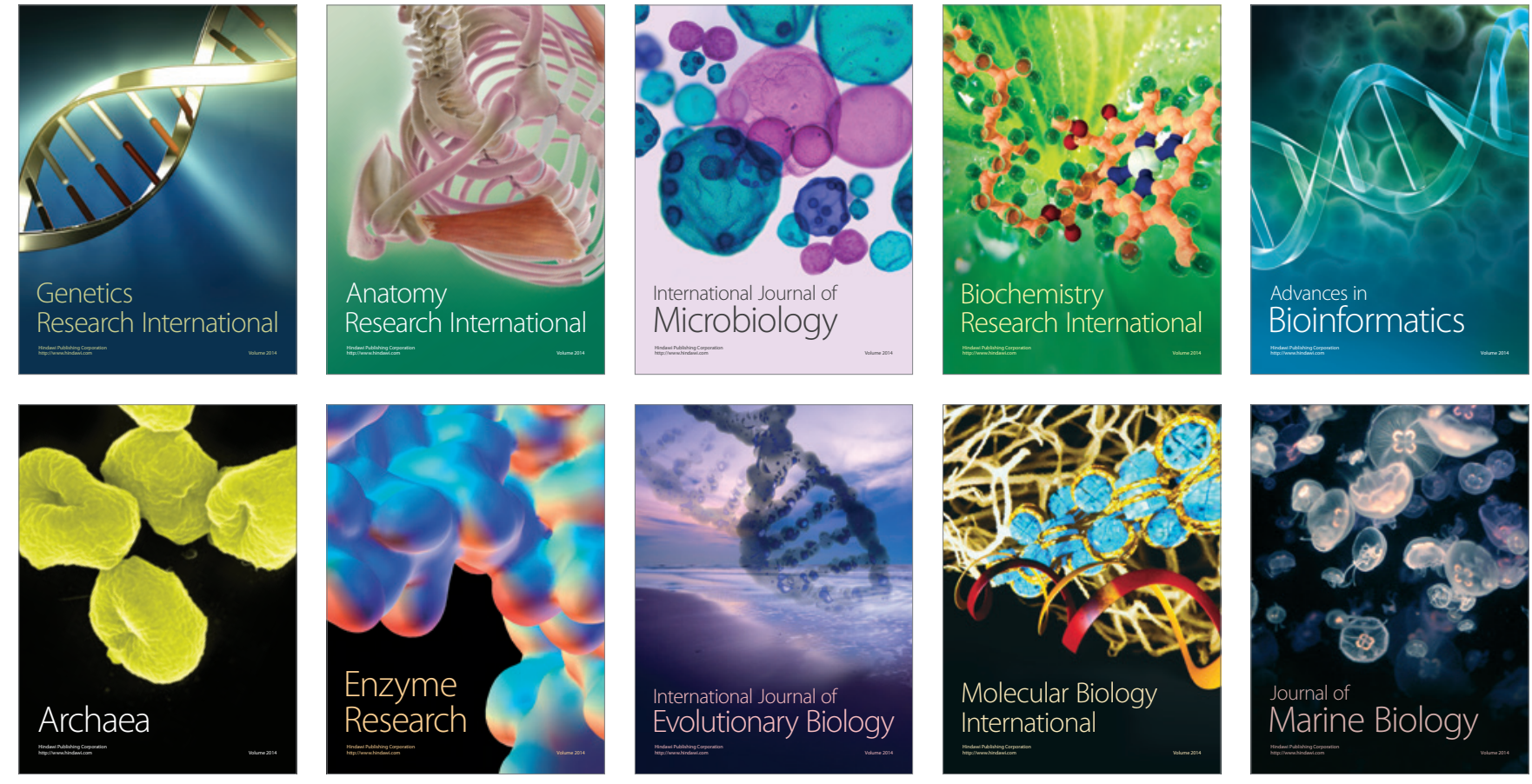